Daniel Schwär*, Andreas Kunz, Stephan Dehen, Frederik Zanger und Fernando Puente León

\title{
Analyse von 3D-CT-Aufnahmen von Spänen zur Extrahierung der Segmentspanbildungsfrequenz
}

\author{
Analysis of 3D-CT-scans of chips for extraction of segmentation frequency
}

DOI 10.1515/teme-2020-0039

Zusammenfassung: Die Vermessung von Spänen, welche bei einem Drehprozess entstehen, ist insbesondere bei einer helixartigen Form eine große Herausforderung. Die Analyse lässt sich nur in den Randbereichen durchführen, da die inneren Bereiche durch den Span selbst verdeckt werden. Außerdem lassen sich Späne, die aus sprödem Material bestehen, nicht zerstörungsfrei analysieren. Da sich metallisches Material sehr genau durch einen Computertomographen vermessen lässt, wurde eine Methode entwickelt, mit welcher sich das 3D-Bild eines Spanes virtuell zu einem geradlinigen Span transformieren lässt. Im Anschluss liegt der Fokus auf der Extrahierung des Segmentspanabstandes, welcher über einen Stauchungsfaktor mit der Segmentspanbildungsfrequenz zusammenhängt. Zuletzt wird die Methode mit einer manuellen Untersuchung der Späne unter einem konfokalen Mikroskop verglichen, wobei gezeigt werden kann, dass der Vorteil der CT-Analyse darin liegt, dass sie auch in der Analyse helixförmiger Späne zu einem Ergebnis kommt.

Schlüsselwörter: Segmentspanbildungsfrequenz, Drehen, Computertomographie.

\begin{abstract}
The analysis of chips produced during a turning process is a difficult challenge, especially with a curled shape. The examination can often only be performed in the peripheral areas, as the inner areas are covered by the chip itself. Chips that consist of brittle material often cannot be analyzed non-destructively. Since metallic material can be measured very accurately by a computer tomograph, a method was developed which makes it possible to transform the $3 \mathrm{D}$ image of a chip virtually into a straight chip. After this, the focus is on the extraction of the chip
\end{abstract}

\footnotetext{
*Korrespondenzautor: Daniel Schwär, Karlsruher Institut für Technologie (KIT), Institut für Industrielle Informationstechnik (IIIT), E-Mail: daniel.schwaer@kit.edu

Andreas Kunz, Fernando Puente León, Karlsruher Institut für Technologie (KIT), Institut für Industrielle Informationstechnik (IIIT)
}

Stephan Dehen, Frederik Zanger, Karlsruher Institut für Technologie (KIT), Institut für Produktionstechnik (wbk) segment length, which is related to the segment frequency by a compression factor. Finally, the method is compared with a manual examination of the chips under a confocal microscope. It can be shown that the CT-analysis comes to a result in the analysis of curled chips.

Keywords: Segmentation frequency, turning, computed tomography.

\section{Einleitung}

Um hochbelastbare Bauteile herzustellen, wie sie beispielsweise in der Luft und Raumfahrt vorkommen [2], werden bestimmte Randschichteigenschaften, insbesondere definierte Eigenspannungszustände, gefordert [9]. In [10] wurde gezeigt, dass die periodisch auftretende Segmentierung des Spanes bei der Zerspanung eine starke Korrelation mit den resultierenden Oberflächeneigenschaften aufweist. Daher ist die Segmentspanbildungsfrequenz eine wichtige Größe bei der Schätzung der Oberflächengüte der Werkstücke. Die Segmentspanbildungsfrequenz hängt bis auf einen Faktor, welcher durch die Stauchung des Spanes bestimmt wird, mit der Segmentlänge zusammen. Dieser kann durch eine Analyse der im Prozess entstandenen Späne bestimmt werden. In [1], [4] und [8] erfolgt die Vermessung der Späne durch optische Untersuchungen. Dies stellt insbesondere bei helixförmigen Spänen ein Problem dar, da der innere Bereich verdeckt ist und sie nur im Randbereich analysiert werden können. Das Zurückbiegen eines helixförmigen Spanes in eine geradlinige Form ist bei spröden Materialien nicht möglich, da diese sehr leicht brechen können.

Metallische Späne können jedoch durch einen 3DComputertomographen (CT) präzise vermessen werden [5]. Basierend darauf wurde eine Methode entwickelt, mit welcher eine dreidimensionale CT-Aufnahme eines Spanes analysiert werden kann. Das Ziel dabei ist es, die Segmentspanbildungsfrequenz zu extrahieren. 


\section{Methode}

Um die Segmentspanbildungsfrequenz zu berechnen muss die Länge der einzelnen Segmente $\Delta L_{s}$ eines Spanes gemessen werden. Die Späne werden allerdings beim Schnitt gestaucht, weswegen, um an die tatsächliche Segmentspanbildungsfrequenz zu kommen, diese Stauchung herausgerechnet werden muss. Wird davon ausgegangen, dass sich das Volumen eines Spanes nicht ändert, lässt sich über die Schnittfläche $A_{0}$, welche sich durch die Zustellung und den Vorschub bestimmen lässt, die Länge des abgetragenen Materials schätzen. Über den Vergleich dieser Länge mit der Spanlänge $L$ kann der Stauchungsfaktor und damit die Segmentspanbildungsfrequenz

$$
f_{s}=\frac{A_{0} \rho L v_{s}}{m_{s} \Delta L_{s}}
$$

berechnet werden. Dabei ist $v_{s}$ die Schnittgeschwindigkeit Das Volumen wird dabei durch die Masse $m$ des Spanes und die Dichte $\rho$ des Materials bestimmt.

Das Ziel der Methode ist es, die Segmentlänge zu bestimmen. Die Grundidee beruht darauf den Span virtuell zu einem geradlinigen Span zu transformieren und anschließend die Ortsfrequenzen des transformierten Spanes zu analysieren.

\subsection{Vorverarbeitung}

Die Daten einer CT-Aufnahme liegen als dreidimensionaler Datenwürfel vor und lassen sich als Matrix $\vec{G}$ mit der Dimension $I \times J \times K$ und den quasi-kontinuierlichen Intensitätswerten $g_{i, j, k}$ interpretieren. Dabei sind $i, j$, und $k$ die Indizes der Matrix. Die Umrechung in die räumlichen Koordinaten erfolgt über

$$
x=i \cdot \Delta x, \quad y=j \cdot \Delta y, \quad \text { und } \quad z=k \cdot \Delta z .
$$

Wobei $\Delta x, \Delta y$ und $\Delta z$ jeweils der Pixelabstand entlang der entsprechenden Koordinatenachse ist.

Um eine Punktwolke aus dem Datenwürfel zu erhalten, muss dieser zunächst einer Binarisierung unterzogen werden. Jeder Punkt muss einer der beiden Klassen „Hintergrund“ (0) oder „Span“ (1) zugewiesen werden. Hierzu wird die K-Means-Methode [7] verwendet. Die Grauwerte der Pixel werden als Punkte im eindimensionalen Raum aufgefasst. Zum Initiieren des K-Means-Algorithmus werden zwei zufällige Klassenschwerpunkte vorgegeben. Die wesentlichen Schritte sind nun das Zuordnen der Punkte zu den beiden Klassen. Ein Punkt wird jeweils der Klasse zugeordnet, deren Klassenschwerpunkt am nächsten liegt. Anschließend werden die Mittelwerte der Punkte einer Klasse berechnet und als neue Klassenschwerpunkte zugewiesen. Diese beiden Schritte werden so lange wiederholt, bis die Klassenschwerpunkte konvergieren. Am Ende kann der Schwellwert mittels dem Durchschnitt der beiden Klassenschwerpunkte berechnet werden.

In dieser Punktwolke kommen teilweise zufällige Punktfehler vor. Um diese Punktfehler herauszufiltern wird das größte zusammenhängende Volumen gesucht. Dazu wird ein „Connected-Component LabelingAlgorithmus" wie er in [3] beschrieben wird, verwendet. Ausgehend von einem initialen Punkt, welcher in der Klasse „Span“ enthalten ist, werden alle angrenzenden Punkte dem ersten Volumen zugeordnet. Werden keine angrenzenden Punkte gefunden, wird ein neuer noch nicht zugeordneter initialer Punkt gewählt und alle angrenzenden Punkte dem nächsten Volumen zugeordnet. Dieser Prozess wird so lange wiederholt, bis alle Punkte einem Volumen zugeordnet sind. Es wird im folgenden das Volumen als Span definiert, welches die meisten zugeordneten Punkte hat. Die Punkte aller anderen Volumina werden der Klasse „Hintergrund" zugeordnet.

\subsection{Räumliche Transformation}

Für die räumliche Transformation wird eine Trajektorie benötigt, welche den Verlauf des Spanes im dreidimensionalen Raum beschreibt. Anschließend sollen einzelne Schnitte des gekrümmten Spanes räumlich zu einem geradlinigen Span transformiert werden. In Abbildung 1 sind die Daten eines typischen Spanes dargestellt. Helixförmige Spanverläufe kommen besonders häufig vor, weswegen die Methodik auf diese Form angepasst wird.

Die Punkte der dreidimensionalen Matrix sind in einem äquidistanten Raster angeordnet und lassen sich in einzelne Tomogramme und damit zweidimensionale Matrizen $\vec{T}_{k}$ entlang der $z$-Achse unterteilen. Die Koordinatenachsen der Matrix werden dabei so definiert, dass die $z$-Achse in Richtung der Rotationsachse der Helixform zeigt.

Für jedes Tomogramm an den Stellen $z_{k}$ lässt sich ein geometrischer Schwerpunkt

$$
\vec{s}\left[z_{k}\right]=\frac{1}{\left|T_{k}\right|} \sum_{\vec{p} \in T_{k}} \vec{p}=\left[\begin{array}{c}
s_{x}\left[z_{k}\right] \\
s_{y}\left[z_{k}\right] \\
z_{k}
\end{array}\right]
$$

des Spanes bestimmen. Die Menge $T_{k}$ enthält dabei alle Punkte der Matrix $\vec{T}_{k}$ mit dem Wert 1. Der Operator $|\cdot|$ bildet die Mächtigkeit der Menge. Der Vektor $\vec{p}=\left[\begin{array}{lll}x & y & z\end{array}\right]$ enthält die dreidimensionalen Koordina- 


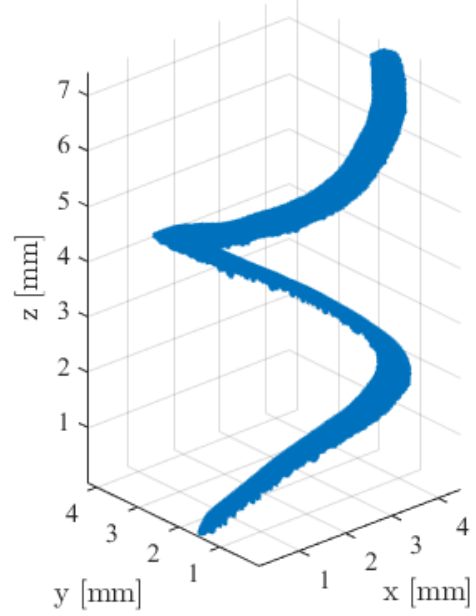

Abb. 1: Beispiel einer binarisierten Punktwolke eines helixförmigen Spanes nach der Entfernung der Punktfehler.

ten eines Punktes. Dieser Schwerpunkt ist ein einfaches Merkmal um den Spanverlauf zu bestimmen.

Da der Verlauf der Schwerpunkte über $z$ im Allgemeinen an einzelnen Stellen Sprünge aufweist, wird der Verlauf der Schwerpunkte mit einer nichtlinearen Filterung geglättet. Hierzu wird sowohl die $x$-, als auch die $y$-Komponente über $z$ aus mehreren benachbarten Schwerpunkten durch ein Polynom $f(z)=a z^{2}+b z+c$ zweiter Ordnung angenähert. Die Parameter des Polynoms lassen sich durch eine Least-Square-Schätzung [6] berechnen. Als Trajektorienpunkt

$$
\vec{t}\left[z_{k}\right]=\left.\left[\begin{array}{c}
a_{x} z^{2}+b_{x} z+c_{x} \\
a_{y} z^{2}+b_{y} z+c_{y} \\
z
\end{array}\right]\right|_{z=z_{k}}
$$

wird jeweils der Punkt $z_{k}$ des approximierenden Polynoms verwendet. Damit ist eine Trajektorie gegeben, die einen glatten Verlauf entlang des Spanes beschreibt.

Für die Berechnung der Segmentlänge ist lediglich die Oberfläche des Spans von Interesse. Damit die Datenmenge reduziert werden kann, wird eine Kantendetektion durchgeführt. Dazu wird jedes Tomogramm $\vec{T}_{k}$ mit einem Sobel-Filter gefiltert und über einen Schwellwert erneut binarisiert.

Der Span kann nun transformiert werden, indem er in mehrere Schnitte, orthogonal zur Trajektorie, unterteilt wird, welche anschließend aneinandergereiht einen geradlinigen Span ergeben. Ein Schnitt wird realisiert, indem an jedem Trajektorienpunkt $\vec{t}[k]$ eine Ebene orthogonal zur Trajektorientangente aufgespannt wird. Die Trajektorie liegt nicht in analytischer Form vor, weswegen die
Tangente und damit die Normale der Ebene durch

$$
\vec{n}\left[z_{k}\right]=\frac{\vec{t}\left[z_{k}+\Delta z\right]-\vec{t}\left[z_{k}\right]}{\left\|\vec{t}\left[z_{k}+\Delta z\right]-\vec{t}\left[z_{k}\right]\right\|}
$$

an jedem Trajektorienpunkt $z_{k}$ geschätzt wird. Für einen Punkt, welcher auf der Seite der Ebene liegt, in die der Normalenvektor zeigt, gilt

$$
\left(\vec{p}-\vec{t}\left[z_{k}\right]\right)^{\mathrm{T}} \vec{n}\left[z_{k}\right]>0 .
$$

Damit können alle Punkte, die zwischen zwei Ebenen liegen, der Menge eines Schnittes zugeordnet werden. Bei helixförmigen Spänen kann es vorkommen, dass in einem Schnitt der beiden Ebenen Punkte aus anderen Spanwindungen mit eingeschlossen werden. Dies kann verhindert werden, indem ein maximaler Radius $r_{\max , z}$ um $\vec{t}\left[z_{k}\right]$ festgelegt wird, welcher die Menge der Punkte eines Schnittes begrenzt. Dieser Radius wird berechnet, indem er iterativ immer größer gewählt wird, bis die Menge der Punkte des Schnittes größer als Null ist und nicht mehr weiter anwächst. Dadurch wird die kleinstmögliche zusammenhängende Oberfläche gefunden. Die Punkte eines Schnittes an der Stelle $z_{k}$ erfüllen die Bedingungen

$$
\begin{aligned}
\left(\vec{p}-\vec{t}\left[z_{k}\right]\right)^{\mathrm{T}} \vec{n}\left[z_{k}\right] & \leq 0, \\
\left(\vec{p}-\vec{t}\left[z_{k}+\Delta z\right]\right)^{\mathrm{T}} \vec{n}\left[z_{k}+\Delta z\right] & >0 \\
\text { und }\left\|\vec{p}-\vec{t}\left[z_{k}\right]\right\| & \leq r_{\max , z}
\end{aligned}
$$

Für jeden Schnitt soll eine räumliche Transformation durchgeführt werden. Der $x-y$-z-Raum wird in den $x^{\prime}-y^{\prime}$ $z^{\prime}$-Raum überführt. Dazu wird jeder Punkt eines Schnittes an der Stelle $z_{k}$ durch

$$
\vec{p}^{\prime}=\vec{K}_{k}\left(\vec{R}_{k}\left(\vec{p}-\vec{t}\left[z_{k}\right]\right)+\vec{l}_{k}\right),
$$

mithilfe der Rotationsmatrizen $\vec{R}_{k}$ und $\vec{K}_{k}$ und der Translationen $\vec{t}\left[z_{k}\right]$ und $\vec{l}_{k}$ transformiert. Die erste Translation um den Trajektorienpunkt $\vec{t}\left[z_{k}\right]$ bildet die Trajektorie auf die neue $z^{\prime}$-Achse ab. Der zweite Translationsvektor

$$
\overrightarrow{l_{k}}=\left[\begin{array}{c}
0 \\
0 \\
\sum_{l=1}^{k}\left\|\vec{t}\left[z_{l-1}\right]-\vec{t}\left[z_{l}\right]\right\|
\end{array}\right]
$$

verschiebt jeden Schnitt in $z^{\prime}$-Richtung an das Ende des Vorherigen, da sonst alle Schnitte im Ursprung bei $z^{\prime}=0$ liegen würden.

Die Rotationsmatrix

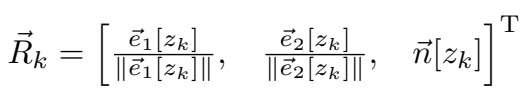

eliminiert die Verdrehung der einzelnen Schnitte, die durch die Helixform des Spanes hervorgerufen wird, und besteht 
aus dem Normalenvektor und den beiden Basisvektoren $\vec{e}_{1}\left[z_{k}\right]$ und $\vec{e}_{2}\left[z_{k}\right]$ der Ebene durch den Trajektorienpunkt $\vec{t}\left[z_{k}\right]$. Für die zwei Basisvektoren verbleibt durch die notwendige Orthogonalitätsbedingung ein Freiheitsgrad. Es wird für $k=0$ ein zufälliges Basisvektorenpaar gewählt und für $k>1$ sukzessiv eine möglichst geringe Aktualisierung vorgenommen. Das bedeutet, dass die beiden anderen Basisvektoren durch

$$
\begin{aligned}
\vec{e}_{1}\left[z_{k}\right] & =\vec{n}\left[z_{k}\right] \times \vec{e}_{2}\left[z_{k-1}\right] \\
\text { und } \quad \vec{e}_{2}\left[z_{k}\right] & =\vec{n}\left[z_{k}\right] \times \vec{e}_{1}\left[z_{k}\right]
\end{aligned}
$$

berechnet werden können. In der Rotationsmatrix werden die normierten Basisvektoren verwendet. Das Nachführen der beiden Basisvektoren hat den Vorteil, dass in den transformierten Schnitten keine Sprünge in der Rotation vorkommen. Es kann lediglich vorkommen, dass sie eine Rotation entlang des Spanverlaufes aufweisen.

Um diese Rotation zu eliminieren kann die typische Form eines Schnittes ausgenutzt werden. Die Oberflächenpunkte eines Schnittes beschreiben meist einen elliptischen Verlauf. Von dieser sollen die Eckpunkte eines jeden Schnittes bestimmt werden. Diese lassen sich ermitteln, indem in jedem Schnitt der euklidische Abstand zwischen allen Extrempunkten $x_{k, \text { min }}^{\prime}, x_{k, \max }^{\prime}, y_{k, \text { min }}^{\prime}$ und $y_{k, \text { max }}^{\prime}$, den äußersten Punkten eines Schnittes in $x^{\prime}$ - und $y^{\prime}$-Richtung, berechnet werden. Es werden die beiden Extrempunkte als Eckpunkte $\vec{\kappa}_{0, k}$ und $\vec{\kappa}_{1, k}$ definiert, die den größten Abstand zueinander aufweisen. Um auch hier mögliche Sprünge zwischen zwei Schnitten zu vermeiden wird dem Eckpunkt der Index 0 zugewiesen, welcher den geringeren euklidischen Abstand zu $\vec{\kappa}_{0, k-1}$ aufweist.

Mithilfe dieser Eckpunkte wird jeder Schnitt um den neuen Ursprung rotiert, sodass der Vektor zwischen den beiden Eckpunkten

$$
\Delta \vec{\kappa}_{k}=\vec{\kappa}_{1, k}-\vec{\kappa}_{0, k}=\left[\begin{array}{ll}
\Delta \kappa_{x, k} & \Delta \kappa_{y, k}
\end{array}\right]^{\mathrm{T}}
$$

entlang der $y^{\prime}$-Achse liegt. Dazu wird die Rotationsmatrix

$$
\vec{K}_{k}=\left[\begin{array}{ccc}
\Delta \kappa_{x, k} & \Delta \kappa_{y, k} & 0 \\
-\Delta \kappa_{y, k} & \Delta \kappa_{x, k} & 0 \\
0 & 0 & 1
\end{array}\right]
$$

verwendet. Somit ist der transformierte Span geradlinig und weist keine Drehung mehr auf. In Abbildung 2 ist ein beispielhafter transformierter Span dargestellt.

\subsection{Analyse der Ortsfrequenz}

Um die Segmentspanbildungsfrequenz zu berechnen, muss der transformierte Span einer Ortsfrequenzanalyse unterzogen werden. Ein Span besitzt in der Regel eine glatte

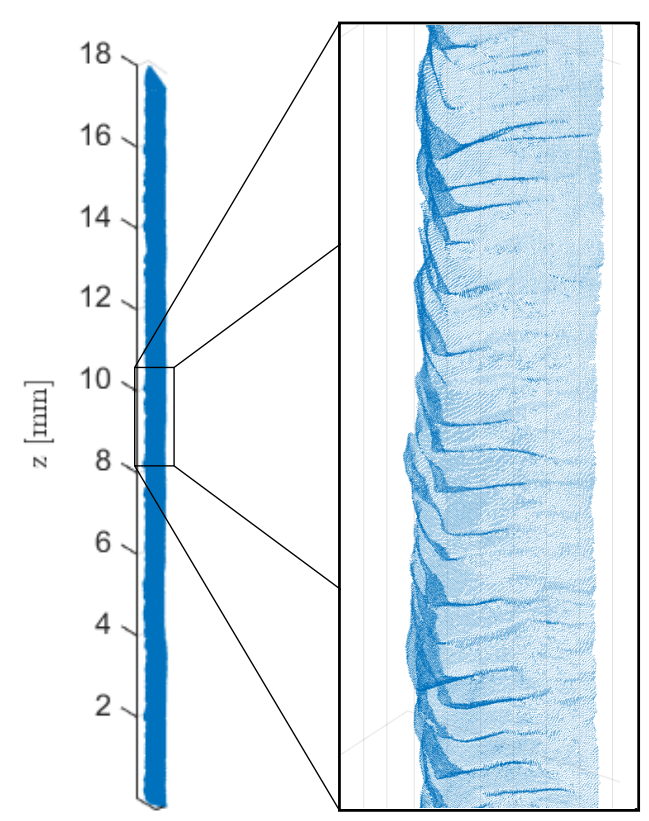

Abb. 2: Ausschnitt aus dem in Abbildung 1 gezeigten Span nach der räumlichen Transformation.

Seite, die an der Werkzeugschneide entstanden ist, und eine Seite, auf der sich die Spansegmente ausbilden. Daher soll die Ortsfrequenzanalyse nur auf einer Seite durchgeführt werden. Hier kann die Beschaffenheit des Spanes ausgenutzt werden, da die Oberfläche von Eckpunkt zu Eckpunkt auf der glatten Seite in der Regel einen kürzeren Verlauf aufweist als auf der Spansegmentenseite. Die Punkte eines Schnittes haben allerdings keine Ordnung. Es ist unklar, ob ein Punkt zu der glatten Seite oder der Spansegmentseite gehört. Dieses Problem kann gelöst werden, indem der Winkel eines Punktes zu dem Durchschnitt $\vec{\mu}_{k}$ aller Punkte eines Schnittes

$$
\alpha_{k, z}=\angle\left(\vec{p}^{\prime}-\vec{\mu}_{k}\right)
$$

berechnet wird. Durch die Kenntnis der Eckpunkte können die beiden Seiten voneinander getrennt werden, indem die Winkel der Eckpunkte als Klassengrenzen verwendet werden. Über die geordneten Punkte können die Pfadlängen beider Seite bestimmt werden. Die längere Seite ist in der Regel die Seite mit den Spansegmenten. Da diese Schätzung jedoch teilweise fehlerhaft ist, wird eine Entscheidung über den gesamten Span getroffen. Es wird die Seite ausgewählt, für welche die Mehrheit der einzelnen Schätzungen spricht.

Für die Ortsfrequenzanalyse wird nun ein Signal benötigt, welches den Oberflächenverlauf entlang einer Geraden in $z^{\prime}$-Richtung beschreibt. Die transformierten Punkte im $x^{\prime}-y^{\prime}-z^{\prime}$-Raum sind allerdings nicht in einem äquidi- 
stanten Raster. Daher wird ein schmales Intervall $\Delta y^{\prime}$ in $y^{\prime}$-Richtung definiert, welches alle Punkte einschließt, die verwendet werden, um das Signal zu erzeugen. Diese werden im Anschluss entsprechend ihrer $z^{\prime}$-Komponente sortiert. Es kommt bei wenigen Spänen durch die Form der Schnitte vor, dass einzelne Punkte der glatten Seite im Signal auftauchen. Diese lassen sich herausfiltern, indem nur Punkte dem Signal zugeordnet werden, die eine $x^{\prime}$-Komponente größer Null aufweisen. Wenn das Intervall $\Delta y^{\prime}$ hinreichend klein gewählt wird, ist der Fehler, welcher durch die nicht identische $y^{\prime}$-Komponente entsteht, sehr gering. Um ein Signal mit äquidistanter Abtastung zu erzeugen, wird eine lineare Interpolation durchgeführt.

Um die Segmentlänge zu schätzen, sollen die Segmente in einem bestimmten Bereich gezählt werden und im Anschluss der mittlere Abstand zwischen diesen Segmenten gemessen werden. Die Segmentanzahl eines Bereiches lässt sich bestimmen, indem die Anzahl der Nulldurchgänge $N_{0}$ des um den Mittelwert befreiten Teilsignales berechnet wird. Da es an einzelnen Stellen des Signals zu hochfrequenten Störungen kommen kann, wird das Signal an äquidistanten Abtastpunkten linear interpoliert und anschließend mit einer gleitenden Mittelung geglättet. Der mittlere Abstand eines Teilsignals $\Delta L$ lässt sich durch die Differenz des ersten und letzten Nulldurchganges schätzen. Die mittlere Segmentlänge kann mit

$$
\Delta L_{s}\left[z^{\prime}\right]=\frac{\Delta L\left[z^{\prime}\right]}{N_{0}\left[z^{\prime}\right]}
$$

bestimmt werden. Die Segmentspanbildungsfrequenz lässt sich mit Gleichung (1) berechnen.

\section{Ergebnisse}

Zur Validierung der Methodik wurde die hier vorgestellte Methode mit den Ergebnissen aus einer manuellen Untersuchung mit einem konfokalen Lichtmikroskops des Typs Nanofocus $\mu$ Soft verglichen. Es wurde ein Objektiv mit einer Vergrößerung von 20 gewählt und die segmentierte Seite des Spanes vermessen. Bei der visuellen Analyse der Daten wurde der Abstand der einzelnen Segmente vermessen und ausgewertet.

Stark helixförmige Späne können mit dem konfokalen Lichtmikroskop nicht untersucht werden, weswegen hier lediglich Ergebnisse für Späne vorliegen, die nur eine leichte Krümmung besitzen. Zum Vergleich der beiden Methoden ist in Tabelle 1 der mittlere Segmentspanabstand für verschiedene Späne dargestellt. Es ist zu erkennen, dass die Werte, die mithilfe des Lichtmikroskops gemessen wurden, von der CT-Spananalyse abweichen. Diese Abweichung

\begin{tabular}{ccc}
\hline & Lichtmikroskop & CT-Spananalyse \\
\hline Span 1 & $90,59 \pm 6,56 \mu \mathrm{m}$ & $98,1125 \pm 4,019 \mu \mathrm{m}$ \\
Span 2 & $93,11 \pm 8,36 \mu \mathrm{m}$ & $108,5701 \pm 11,148 \mu \mathrm{m}$ \\
Span 3 & $86,12 \pm 5,48 \mu \mathrm{m}$ & $95,2018 \pm 13,609 \mu \mathrm{m}$ \\
Span 4 & - & $74,1022 \pm 17,086 \mu \mathrm{m}$ \\
Span 5 & - & $118,6926 \pm 14,310 \mu \mathrm{m}$ \\
Span 6 & - & $97,5164 \pm 12,221 \mu \mathrm{m}$ \\
\hline
\end{tabular}

Tab. 1: Vergleich der CT-Spanauswertung mit der Untersuchung durch ein konfokales Lichtmikroskop.

kann daher kommen, dass die Untersuchung mithilfe des Lichtmikroskops meist an Stellen mit stark ausgeprägten Segmenten durchgeführt wird, wohingegen die CTSpananalyse Messwerte über den gesamten Spanverlauf liefert. An Span 4 bis 6 ist außerdem ersichtlich, dass die CT-Spananalyse auch eine Schätzung des Segmentabstandes bei helixförmigen Spänen ermöglicht, wohingegen die Untersuchung mittels Lichtmikroskop hier kein Ergebnis liefern kann.

In Abbildung 3 ist die Schätzung des Segmentabstandsverlaufes entlang des Spanes 5, welcher beispielhaft in Abbildung 1 und 2 dargestellt wurde, dargestellt. Es

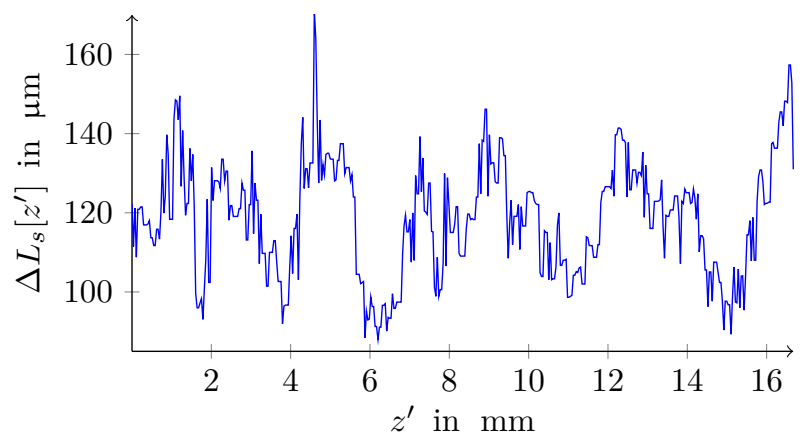

Abb. 3: Mittlere Segmentlänge $\Delta L_{s}\left[z^{\prime}\right]$ über den Spanverlauf in $z^{\prime}$-Richtung von Span 5.

lässt sich erkennen, dass die Auswertung im Vergleich mit den anderen Spänen eine hohe Varianz ausweist. Diese Varianz hängt damit zusammen, dass die Segmente des Spanes in manchen Bereichen nicht besonders stark ausgeprägt sind. Es lassen sich selbst bei manueller Überprüfung, die Segmente teilweise nicht sicher erkennen. Im Gegensatz dazu ist in Abbildung 4 die Schätzung des Segmentlängenverlaufes von Span 1 dargestellt. Dieser Span weist eine deutlichere Segmentierung auf. Diese deutlichere Segmentierung hat eine geringere Varianz zur Folge. 


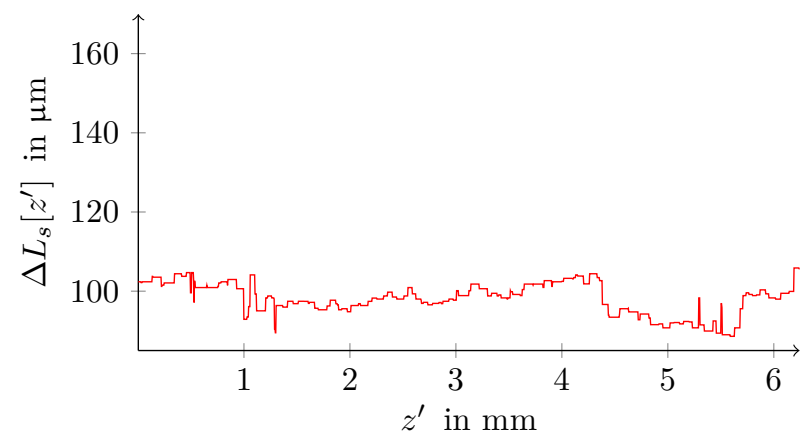

Abb. 4: Mittlere Segmentlänge $\Delta L_{s}\left[z^{\prime}\right]$ über den Spanverlauf in $z^{\prime}$-Richtung von Span 1.

\section{Zusammenfassung}

Es wurde eine Methode vorgestellt, mit der 3D-CTAufnahmen von Metallspänen aus Schnittprozessen untersucht werden können. Dazu werden die Aufnahmen in einem ersten Schritt durch eine Schwellwertfilterung binarisiert. Die Auswahl des Schwellwertes kann durch den kMeans-Algorithmus realisiert werden. Anschließend wird, um einzelne Punktfehler zu eliminieren, ein „ConnectedComponent Labeling-Algorithmus" angewandt, welcher das größte zusammenhängende Volumen zu bestimmt. In den so vorverarbeiteten Daten wird für jedes einzelne Tomogramm der Schwerpunkt berechnet. Diese Schwerpunkte werden durch eine Filterung geglättet und bilden damit eine Trajektorie, die den Verlauf des Spanes beschreibt. Entlang dieser Trajektorie wird der Span in einzelne Schnitte unterteilt, welche aneinandergehängt und gedreht einen geradlinigen Span ergeben. Anhand der Oberflächenpunkte entlang einer Geraden wird im Anschluss die Segmentlänge vermessen. Dazu wird der Oberflächenverlauf in kleinere Abschnitte unterteilt und die Nulldurchgänge gezählt. Durch den Abstand des ersten und letzten Nulldurchganges eines Teilsignals kann die mittlere Segmentlänge bestimmt werden. Unter der Annahme, dass sich das abgetragene Volumen bei der Entstehung des Spanes nicht ändert, kann über das Spangewicht und die Länge der Stauchungsfaktor bestimmt werden, mit dem der Segmentspanabstand in die Segmentspanbildungsfrequenz umgerechnet werden kann. Es konnte gezeigt werden, dass mithilfe der CT-Spananalyse, im Gegensatz zur manuellen Untersuchung mit einem Lichtmikroskop, die Analyse von helixförmigen Spänen möglich ist.

\section{Literatur}

[1] J. Barry, G. Byrne und D. Lennon. Observations on chip formation and acoustic emission in machining ti-6al-4v alloy. International Journal of Machine Tools and Manufacture, 41 (7):1055-1070, 2001.

[2] R. Boyer. An overview on the use of titanium in the aerospace industry. Materials Science and Engineering: A, 213(1): $103-114,1996$. International Symposium on Metallurgy and Technology of Titanium Alloys.

[3] A. S. Chowdhury. Computer vision-guided virtual craniofacial surgery: A graph-theoretic and statistical perspective, 2011.

[4] M. Cotterell und G. Byrne. Characterisation of chip formation during orthogonal cutting of titanium alloy ti-6al-4v. CIRP Journal of Manufacturing Science and Technology, 1(2): 81-85, 2008.

[5] A. Devotta, T. Beno, R. Löf und E. Espes. Quantitative characterization of chip morphology using computed tomography in orthogonal turning process. Procedia CIRP, 33(1): 299-304, 2015.

[6] F. P. León und U. Kiencke. Messtechnik, Band 10. Springer, 2015.

[7] D. Liu und J. Yu. Otsu method and k-means. In 2009 Ninth International Conference on Hybrid Intelligent Systems,

Band 1, S. 344-349, 2009.

[8] V. Nguyen, P. Fernandez-Zelaia und S. N. Melkote. Pvdf sensor based characterization of chip segmentation in cutting of ti-6al-4v alloy. CIRP Annals, 66(1):73-76, 2017.

[9] F. Zanger. Segmentspanbildung, Werkzeugverschleiß, Randschichtzustand und Bauteileigenschaften: Numerische Analysen zur Optimierung des Zerspanungsprozesses am Beispiel von Ti-6Al-4V. PhD thesis, 2012.

[10] F. Zanger, A. Kacaras, M. Bächle, M. Schwabe, F. P. León und $V$. Schulze. Fem simulation and acoustic emission based characterization of chip segmentation frequency in machining of ti-6al-4v. Procedia CIRP, 72:1421-1426, 2018. 